\title{
Partitions and Compositions Defined by Inequalities
}

\author{
Sylvie Corteel \\ CNRS PRiSM, UVSQ \\ 45 Avenue des Etats-Unis \\ 78035 Versailles, France \\ syl@prism.uvsq. fr
}

\author{
Carla D. Savage * \\ Dept. of Computer Science \\ N. C. State University, Box 8206 \\ Raleigh, NC 27695, USA \\ savage@csc.ncsu.edu
}

October 26, 2018

\begin{abstract}
We consider sequences of integers $\left(\lambda_{1}, \ldots, \lambda_{k}\right)$ defined by a system of linear inequalities $\lambda_{i} \geq \sum_{j>i} a_{i j} \lambda_{j}$ with integer coefficients. We show that when the constraints are strong enough to guarantee that all $\lambda_{i}$ are nonnegative, the generating function for the integer solutions of weight $n$ has a finite product form $\prod_{i}\left(1-q^{b_{i}}\right)^{-1}$, where the $b_{i}$ are positive integers that can be computed from the coefficients of the inequalities. The results are proved bijectively and are used to give several examples of interesting identities for integer partitions and compositions. The method can be adapted to accommodate equalities along with inequalities and can be used to obtain multivariate forms of the generating function. We show how to extend the technique to obtain the generating function when the coefficients $a_{i, i+1}$ are allowed to be rational, generalizing the case of lecture hall partitions. Our initial results were conjectured thanks to the Omega package [6].
\end{abstract}

\section{Introduction}

For a sequence $\lambda=\left(\lambda_{1}, \lambda_{2}, \ldots, \lambda_{k}\right)$ of integers, define the weight of $\lambda$ to be $\lambda_{1}+\cdots+\lambda_{k}$ and call each $\lambda_{i}$ a part of $\lambda$. If a sequence $\lambda$ of weight $n$ has all parts nonnegative, we call it a composition of $n$ into $k$ nonnegative parts and if, in addition, $\lambda$ is a nonincreasing sequence, we call it a partition of $n$ into at most $k$ parts. In the sequel we will consider that $\lambda_{i}=0$ if $i<0$ or $i>k$.

In this paper we study partitions and compositions into $k$ nonnegative parts defined by equalities and inequalities. This work was motivated by results of the form :

- Given a positive integer $r$, the partitions $\lambda=\lambda_{1}, \ldots, \lambda_{k}$ of $n$ which satisfy $\lambda_{i} \geq r \lambda_{i+1}$ for $1 \leq i \leq k$ have weight generating function $\prod_{i=0}^{k-1}\left(1-q^{1+r+\cdots+r^{i}}\right)^{-1}[14$.

* Research supported by NSA grants MDA 904-00-1-0059 and MDA 904-01-0-0083 
- Given a positive integer $r$ the generating function of the partitions $\lambda$ of $n$ with at most $k$ parts and $\lambda_{i} \geq \sum_{j=1}^{r}(-1)^{j+1}\left(\begin{array}{c}r \\ j\end{array}\right) \lambda_{j+i}, 1 \leq i<k$ is : $\prod_{i=0}^{k-1}\left(1-q^{\left(\begin{array}{c}i+r \\ r\end{array}\right)}\right)^{-1}$. See 2, 12, 19].

- The generating function of the partitions $\lambda$ of $n$ with at most $k$ parts and $\lambda_{i} /(k-i+$ 1) $\geq \lambda_{i+1} /(k-i), 1 \leq i<k$ is : $\prod_{i=1}^{k}\left(1-q^{2 i-1}\right)^{-1}$. See the Lecture Hall Theorem in [10].

More generally, we consider integer sequences $\lambda$ of length $k$ satisfying $\lambda_{i} \geq \sum_{j=i+1}^{k} a_{i j} \lambda_{i}$ where the $a_{i j}$ guarantee that all $\lambda_{i} \geq 0$. We show in Section 2 that when the $a_{i j}$ are all integers, the generating function for these compositions is $\prod_{i=1}^{k}\left(1-q^{b_{i}}\right)^{-1}$, where $b=$ $\left(b_{1}, \ldots, b_{k}\right)$ is a sequence of positive integers. Several generalizations are included.

In Section 3, we consider rational coefficients $a_{i j}$. We show how to use the results of Section 2 to give an explicit form for the generating function for any set of compositions defined by the ratio of consecutive parts:

$$
\lambda_{1} \geq \frac{n_{1}}{d_{1}} \lambda_{2} ; \quad \lambda_{2} \geq \frac{n_{2}}{d_{2}} \lambda_{3} ; \quad \lambda_{3} \geq \frac{n_{3}}{d_{3}} \lambda_{4} ; \quad \ldots ; \quad \lambda_{k-1} \geq \frac{n_{k-1}}{d_{k-1}} \lambda_{k} ; \quad \lambda_{k} \geq 0 .
$$

This result has been implemented in Maple and our experiments have led to several interesting results. In Section 3.2 we focus on one special case which has consequences such as: the compositions of $n$ into at most $k$ parts satisfying

$$
\lambda_{1} \geq \lambda_{2} \geq \lambda_{3} / 2 \geq \lambda_{4} \geq \lambda_{5} / 2 \geq \cdots \geq \lambda_{2 k} \geq \lambda_{2 k+1} / 2 \geq 0
$$

are in one-to-one correspondence with the set of partitions of $n$ into parts of size at most $3 k+2$ in which parts divisible by 3 can appear at most once.

In [10], Bousquet-Mélou and Eriksson considered the set $L_{k}$ of partitions $\lambda$ into at most $k$ parts satisfying $\lambda_{i} /(k-i+1) \geq \lambda_{i+1} /(k-i)$, for $1 \leq i<k$, and proved the following Lecture Hall Theorem:

$$
\sum_{\lambda \in L_{k}} q^{|\lambda|}=\prod_{i=1}^{k} \frac{1}{1-q^{2 i-1}} .
$$

In Section 4, we study the 2-variable generating function and show a slight generalization of (11) in which the constraints on $\lambda_{1}$ can be modified.

Our initial results were conjectured thanks to experiments with the Omega package [6. 4], a Mathematica implementation of the Omega operator defined by MacMahon [15]. This operator was then not used for 85 years except by Stanley in 1973 [16]. A few years ago Andrews revived this operator [1, 2] and used it in [1] to give a second proof of the Lecture Hall Theorem. In conjunction with Paule and Riese, he implemented the operator in the Omega package and together they have continued to identify the power of the Omega operator for such combinatorial problems as magic squares [9], hypergeometric multisums [3], constrained compositions [8], plane partitions diamonds [5], and $k$-gons partitions [7]. 


\section{Integer Coefficients}

\subsection{Overview}

A matrix $A$ is called upper triangular if $A[i, j]=0$ for $i>j$ and strictly upper triangular if $A[i, j]=0$ for $i \geq j$.

Let $A[1 . . k, 1 . . k]$ be a strictly upper triangular matrix of integers and let $P_{A}$ be the set of sequences $\lambda=\left(\lambda_{1}, \lambda_{2}, \ldots, \lambda_{k}\right)$ satisfying

$$
\lambda_{i} \geq \sum_{j=i+1}^{k} A[i, j] \lambda_{j} \quad \text { for } 1 \leq i \leq k .
$$

In this section we show that if every $\lambda \in P_{A}$ is a sequence of nonnegative integers, that is, a composition, then the generating function for the elements of $P_{A}$ of weight $n$ is

$$
\prod_{i=1}^{k} \frac{1}{1-q^{b_{i}}}
$$

where $\left\{b_{i}\right\}$ is sequence of positive integers which can be computed from $A$.

\subsection{The Details}

Given $A[1 . . k, 1 . . k]$, a strictly upper triangular matrix of integers, we know $A$ is nilpotent, that is, $A^{k}=0$. Considering $\lambda$ as a column vector, the matrix inequality, $\lambda \geq A \lambda$, describes the solutions to (2). Then

$$
\lambda=A \lambda+\left[s_{1}, s_{2}, \ldots s_{k}\right]^{T}
$$

where $s=\left(s_{1}, \ldots, s_{k}\right)$ is the column vector of nonnegative integers defined by

$$
s_{i}=\lambda_{i}-\sum_{j=i+1}^{k} A[i, j] \lambda_{j} .
$$

Iterating (3) yields

$$
\lambda=\left(I+A+A^{2}+\cdots+A^{k-1}\right)\left[s_{1}, s_{2}, \ldots s_{k}\right]^{T}
$$

since $A^{k}=0$. Since $(I-A)\left(I+A+A^{2}+\cdots+A^{k-1}\right)=I, I-A$ is invertible, and its inverse is an upper triangular matrix of integers, since $A$ is. Thus,

$$
\lambda=(I-A)^{-1}\left[s_{1}, s_{2}, \ldots s_{k}\right]^{T} .
$$

Let $B=(I-A)^{-1}$. Then

$$
P_{A}=\left\{B\left[s_{1}, \ldots, s_{k}\right]^{T} \mid s_{i} \geq 0 \text { for } 1 \leq i \leq k\right\} .
$$


Lemma 1 The set $P_{A}$, of solutions to (2), is a set of compositions if and only if every element of $B=(I-A)^{-1}$ is nonnegative.

Proof If $\lambda \in P_{A}$, then from (44), $\lambda=B\left[s_{1}, \ldots, s_{k}\right]^{T}$ for some sequence of nonnegative integers $\left\{s_{i}\right\}$. If all elements of $B$ are nonnegative, $\lambda_{i} \geq 0$ for $1 \leq i \leq k$. However, if some entry $B[j, l]$ is negative, then choosing $\left\{s_{i}\right\}$ so that $s_{l}=1$ and $s_{i}=0$ if $i \neq l$ makes entry $\lambda_{j}$ of $\lambda=B\left[s_{1}, \ldots, s_{k}\right]^{T}$ negative.

Theorem 1 Let $P_{A}(n, k)$ be the set of sequences $\lambda=\left(\lambda_{1}, \lambda_{2}, \ldots, \lambda_{k}\right)$ of weight $n$ satisfying

$$
\lambda_{i} \geq \sum_{j=i+1}^{k} A[i, j] \lambda_{j} \quad \text { for } 1 \leq i \leq k .
$$

If every $\lambda \in P_{A}$ is a composition, then the generating function for $P_{A}(n, k)$ is

$$
\sum_{n=0}^{\infty}\left|P_{A}(n, k)\right| q^{n}=\prod_{i=1}^{k} \frac{1}{1-q^{b_{i}}},
$$

where $b_{i}=\sum_{j} B[j, i]$ and $B=(I-A)^{-1}$.

Proof Let $h=[1,1, \ldots, 1]$ be the row vector of length $k$ containing only ones. The weight of a composition $\lambda \in P_{A}$ is $|\lambda|=h \lambda=h B\left[s_{1}, \ldots s_{k}\right]^{T}$, using (44), where $s_{i}=$ $\lambda_{i}-\sum_{j=i+1}^{k} A[i, j] \lambda_{j}$. Define $b=h B$. Then $b_{i}=\sum_{j} B[j, i]$ and the weight generating function of $P_{A}$ is

$$
\sum_{\lambda \in P_{A}} q^{\lambda_{1}+\cdots+\lambda_{k}}=\sum_{s_{1}, \ldots, s_{k} \geq 0} q^{b_{1} s_{1}+\cdots+b_{k} s_{k}}=\prod_{i=1}^{k} \frac{1}{1-q^{b_{i}}} .
$$

Note that this argument establishes that the mapping

$$
\Theta(\lambda)=\left(b_{1} s_{1}, \ldots, b_{k} s_{k}\right)
$$

where

$$
s_{i}=\lambda_{i}-\sum_{j=i+1}^{k} A[i, j] \lambda_{j}
$$

is a bijection from $P_{A}(n, k)$ to the set of sequences $r_{1}, \ldots, r_{k}$ of weight $n$ in which $r_{i}$ is a nonnegative multiple of $b_{i}$. In the case that the $b_{i}$ are distinct positive integers, this can be viewed alternatively as a bijection with partitions of $n$ into parts in $\left\{b_{1}, \ldots, b_{k}\right\}$.

Example 0 Starting with the matrix

$$
A=\left[\begin{array}{cccc}
0 & 2 & -1 & 0 \\
0 & 0 & 2 & -1 \\
0 & 0 & 0 & 2 \\
0 & 0 & 0 & 0
\end{array}\right] \quad \text { we get } \quad B=(I-A)^{-1}=\left[\begin{array}{cccc}
1 & 2 & 3 & 4 \\
0 & 1 & 2 & 3 \\
0 & 0 & 1 & 2 \\
0 & 0 & 0 & 1
\end{array}\right]
$$


and summing each column of $B$ gives the sequence $b=(1,3,6,10)$. By Theorem 1 the generating function for $P_{A}$ is $\left[(1-q)\left(1-q^{3}\right)\left(1-q^{6}\right)\left(1-q^{10}\right)\right]^{-1}$.

If $A$ is a strictly upper triangular matrix of integers with the property that $P_{A}$ is a set of partitions and if the $b_{i}$ are distinct positive integers, Theorem 1 gives a bijection between two sets of partitions. Several such sample results, including Example 1 below, appear throughout this section. In other situations, such as Example 2 below, Theorem 1 gives a bijection between families of compositions. Later examples, such as Example 15 in Section 3 , will show bijections between a family of compositions, on the one hand, and a family of partitions on the other.

Example 1 The partitions $\lambda$ of $n$ with at most $k$ parts and with $\lambda_{1} \geq \sum_{i=2}^{k} \lambda_{i}$ comprise $P_{A}$ where, $A[i, j]=1$ if $1=i<j$ or $j=i+1$ and $A[i, j]=0$ otherwise. Then the matrix $B=(I-A)^{-1}$ is:

$$
B[i, j]= \begin{cases}0 & \text { if } j<i \\ 1 & \text { if } j=i \text { or } j>i>1 \\ j-1 & \text { otherwise. }\end{cases}
$$

Summing the $i$ th column in the matrix $B=(I-A)^{-1}$ gives $b_{i}=2(i-1)$ for $2 \leq i \leq k$ and $b_{1}=1$, so by Theorem 1 , the generating function is

$$
(1-q)^{-1} \prod_{i=1}^{k-1}\left(1-q^{2 i}\right)^{-1}
$$

Let $P_{A,=}(n, k)$ be the set of compositions of $n$ in $P_{A}$ with at most $k$ parts and with $\lambda_{1}=\sum_{j=2}^{k} A[i, j] \lambda_{j}$. Since this forces $s_{0}=0$ in (44), we get the following from 6 .

Corollary 1 Let $P_{A,=}(n, k)$ be the set of compositions in $P_{A}$ of $n$ with at most $k$ parts and with $\lambda_{1}=\sum_{j=2}^{k} A[i, j] \lambda_{j}$. The generating function for $P_{A,=}(n, k)$ is

$$
\prod_{i=2}^{k} \frac{1}{1-q^{b_{i}}}
$$

Example 2 There is a one-to-one correspondence between sequences $\lambda_{1}, \ldots, \lambda_{k}$ of weight $n$ satisfying

$$
\begin{aligned}
& \sum_{j=0}^{k-1}(-1)^{j} \lambda_{1+j}=0 \\
& \sum_{j=0}^{k-i}(-1)^{j} \lambda_{i+j} \geq 0 \quad \text { for } 2 \leq i \leq k
\end{aligned}
$$

and the set of compositions of $n$ into $k-1$ even parts. The $\lambda$ satisfying the constraints are compositions and they comprise the set $P_{A}(n, k)$ where $A$ is the $k \times k$ upper triangular 
matrix defined by $A[i, i]=0$ and $A[i, j]=(-1)^{i+j+1}$. The result follows from Corollary 1 by summing the columns of $B=(I-A)^{-1}$ to get $b_{2}=b_{3}=\cdots=b_{k}=2$.

Example 3 The generating function of the partitions $\lambda$ of $n$ with at most $k$ parts and with $\lambda_{1} \geq 2 \lambda_{2}+\sum_{i=2}^{k-1} \lambda_{i+1}$ and $\lambda_{2} \geq \sum_{i=2}^{k-1} \lambda_{i+1}$ is :

$$
\frac{1}{(1-q)\left(1-q^{3}\right)} \prod_{i=1}^{k-2} \frac{1}{1-q^{5 i}} .
$$

This follows from Theorem 11 Since $\lambda$ is a partition, for $i>2, A[i, j]=1$ when $j=i+1$ and 0 otherwise. When $i \leq 2, A[1,2]=2$ and otherwise $A[i, j]=1$ for $i=1,2, j>i$, so $b_{1}=1, b_{2}=3$, and $b_{i}=5(i-2)$ for $3 \leq i \leq k$.

\subsection{Further Examples and Generalizations}

Theorem 1] gives bijective proofs of identities such as the two first examples given in the introduction.

Example 4 Given a positive integer $r$, the partitions $\lambda=\lambda_{1}, \ldots, \lambda_{k}$ of $n$ which satisfy $\lambda_{i} \geq r \lambda_{i+1}$ for $1 \leq i \leq k-1$ correspond to the constraint matrix $A$ with $A[i, i+1]=r$. Then $B$ has $B[i, j]=r^{j-i}, j \geq i$, and 0 otherwise, so by Theorem 1 the generating function is $\prod_{i=0}^{k-1}\left(1-q^{1+r+\cdots+r^{i}}\right)^{-1}$. See [14].

Example 5 Given $r$, a positive integer. Consider the partitions $\lambda$ of $n$ with at most $k$ parts and $\lambda_{i} \geq \sum_{j=1}^{r}(-1)^{j+1}\left(\begin{array}{c}r \\ j\end{array}\right) \lambda_{j+i}$, that is : $A[i, j]=\left\{\begin{array}{ll}0 & \text { if } i \leq j \\ (-1)^{j-i+1}\left(\begin{array}{c}r \\ j-i\end{array}\right) & \text { otherwise }\end{array}\right.$. The generating function is

$$
\prod_{i=0}^{k-1} \frac{1}{1-q^{\left(\begin{array}{c}
r+i \\
r
\end{array}\right)}}
$$

since it can be shown that $B[i, j]=\left\{\begin{array}{ll}0 & \text { if } i>j \\ \left(\begin{array}{c}r-1+j-i \\ r-1\end{array}\right) & \text { otherwise }\end{array}\right.$. See [2, 12].

Example 6 The generating function of the partitions $\lambda$ of $n$ with at most $k$ parts and $\lambda_{i} \geq \lambda_{i+1}+\lambda_{i+2}, 1 \leq i \leq k$ is : $\prod_{i=2}^{k+1}\left(1-q^{F_{i}-1}\right)^{-1}$, where $F_{i}$ is the $i^{\text {th }}$ Fibonacci number (defined by $F_{0}=F_{1}=1$ and $F_{i}=F_{i-1}+F_{i-2}$ for $i>1$.) In this case, $B[i, j]=F_{j-i}$ if $j \geq i$ and 0 otherwise.

Example 7 Studying partitions with $\lambda_{i} \geq i \sum_{j=i+1}^{k} \lambda_{j}, 1 \leq i \leq k$, we get $B[i, j]=1$ if $i=j, j ! /((i+1)(i-1) !)$ if $i<j$ and 0 otherwise. It is easy to show by induction that

$$
\sum_{i=1}^{j-1} \frac{1}{(i+1)(i-1) !}=\frac{j !-1}{j !} .
$$

Hence $b_{j}=\sum_{i} B[j, i]=1+\sum_{i=1}^{j-1} \frac{j !}{(i+1)(i-1) !}=j$ !. The generating function of the partitions $\lambda$ of $n$ with at most $k$ parts and $\lambda_{i} \geq i \sum_{j=i+1}^{k} \lambda_{j}, 1 \leq i \leq k$, is : $\prod_{j=1}^{k}\left(1-q^{j !}\right)^{-1}$. 
We can generalize Theorem 1 to allow the constraints of the matrix $A$ to be satisfied with equality for any specified set of $\lambda_{i}$.

Corollary 2 Given a set $S \subseteq\{1,2, \ldots, k\}$, let $P_{A}(n, k ; S)$ be the set of sequences $\lambda=$ $\left(\lambda_{1}, \lambda_{2}, \ldots, \lambda_{k}\right)$ of weight $n$ satisfying, for $1 \leq i \leq k$ :

$$
\begin{gathered}
\lambda_{i}=\sum_{j=i+1}^{k} A[i, j] \lambda_{j} \quad \text { if } i \in S \\
\lambda_{i} \geq \sum_{j=i+1}^{k} A[i, j] \lambda_{j} \quad \text { if } i \notin S .
\end{gathered}
$$

If all elements of $P_{A}$ are compositions, the generating function for $P_{A}(n, k ; S)$ is

$$
\sum_{n=0}^{\infty}\left|P_{A}(n, k ; S)\right| q^{n}=\prod_{i=1, i \notin S}^{k} \frac{1}{1-q^{b_{i}}},
$$

where the $b_{i}=\sum_{j} B[j, i]$.

\section{Proof}

For $1 \leq i \leq k$, let

$$
s_{i}=\lambda_{i}-\sum_{j=i+1}^{k} A[i, j] \lambda_{j} .
$$

Any $\lambda \in P_{A}(n, k ; S)$ must have $s_{i}=0$ for $i \in S$, so the result follows from (6) in the proof of Theorem 1

Example 8 Consider the set of partitions $\lambda$ of $n$ with at most $k$ parts and with $\lambda_{1}=\sum_{i=2}^{k} \lambda_{i}$ and $\lambda_{2}=\sum_{i=3}^{k} \lambda_{i}$. Here $S=\{1,2\}, b_{1}=1, b_{2}=2$, and $b_{i}=4(i-2)$ for $3 \leq i \leq k$, so by Corollary 2 , the generating function is

$$
\prod_{i=1}^{k-2}\left(1-q^{4 i}\right)^{-1}
$$

Corollary 3 Given a set $S \subseteq\{1, \ldots k\}$ and nonnegative integers $d_{1}, d_{2}, \ldots d_{k}$, let $D_{A}(n, k ; S)$ be the set of sequences $\lambda=\left(\lambda_{1}, \lambda_{2}, \ldots, \lambda_{k}\right)$ of weight $n$ into nonnegative parts satisfying, for $1 \leq i \leq k$ :

$$
\begin{aligned}
& \lambda_{i}=\sum_{j=i+1}^{k} A[i, j] \lambda_{j}+d_{i} \quad \text { if } i \in S \\
& \lambda_{i} \geq \sum_{j=i+1}^{k} A[i, j] \lambda_{j}+d_{i} \quad \text { if } i \notin S .
\end{aligned}
$$


If all elements of $P_{A}$ are compositions, the generating function for $D_{A}(n, k ; S)$ is just

$$
\prod_{i=1}^{k} q^{d_{i} b_{i}} \sum_{n=0}^{\infty}\left|P_{A}(n, k ; S)\right| q^{n}
$$

Proof In this case, (3) becomes $\lambda \geq A \lambda+\left[s_{1}+d_{1}, \ldots, s_{k}+d_{k}\right]^{T}$, where $s_{i}=0$ when $i \in S$. By (6) in the proof of Theorem 10 the generating function becomes

$$
\sum_{\lambda \in D_{A}(n, k ; S)} q^{\lambda_{1}+\cdots+\lambda_{k}}=\sum_{s_{1}, \ldots, s_{k} \geq 0} q^{b_{1}\left(s_{1}+d_{1}\right)+\cdots+b_{k}\left(s_{k}+d_{k}\right)}=\prod_{i=1}^{k} q^{d_{i} b_{i}} \sum_{n=0}^{\infty}\left|P_{A}(n, k ; S)\right| q^{n} .
$$

Example 9 Consider the partitions $\lambda_{1}, \ldots, \lambda_{k}$ of $n$ satisfying $\lambda_{1}=\lambda_{2}+\sum_{i=4}^{k} \lambda_{i}$ or $\lambda_{1}=$ $\lambda_{2}+\sum_{i=4}^{k} \lambda_{i}+1$. These are in one-to-one correspondence with the partitions of $n$ into odd parts of size at most $2 k-3$. To see this, note that for both sets of constraints, $S=\{1\}$, $b_{1}=1, b_{2}=2$ and $b_{i}=2 i-3$ for $3 \leq i \leq k$. For the first set, all $d_{i}=0$. For the second set, $d_{1}=1$, and $d_{i}=0$ for $i>1$. So by Corollary 3 , the generating function is:

$$
\frac{1+q}{\left(1-q^{2}\right)} \prod_{i=3}^{k-1} \frac{1}{\left(1-q^{2 i-3}\right)}=\prod_{i=1}^{k-1} \frac{1}{\left(1-q^{2 i-1}\right)}
$$

Example 10 Partitions of $n$ into $k$ odd parts can be viewed as those sequences $\left(\lambda_{1}, \ldots, \lambda_{2 k}\right)$ satisfying $\lambda_{2 k} \geq 0$ and for $i<2 k$,

$$
\lambda_{i}=\lambda_{i+1}+1 \quad \text { if } i \text { is odd; } \quad \lambda_{i} \geq \lambda_{i+2} \quad \text { if } i \text { is even. }
$$

(Just let $\alpha_{i}=\lambda_{2 i-1}+\lambda_{2 i}$.) For this system, $d_{i}=1$ if $i$ is odd and 0 if $i$ is even and $S=\{1,3,5, \ldots, 2 k-1\}$. So, summing the columns of $B=I-A^{-1}$, we get $b_{i}=1$ if $i$ is odd and $i$ if $i$ is even and by Corollary 3, the generating function is

$$
q^{k} \prod_{i=1}^{k}\left(1-q^{2 i}\right)^{-1}
$$

giving (not surprisingly) a bijection with partitions of $n-k$ into $k$ nonnegative even parts.

Remark Note that we can link these results to partition analysis. The Omega operator $\Omega$
$\geq[15$ is defined as follows :

$$
\Omega \sum_{s_{1}=-\infty}^{\infty} \ldots \sum_{s_{r}=-\infty}^{\infty} A_{s_{1}, \ldots, s_{r}} \omega_{1}^{s_{1}} \ldots \omega_{r}^{s_{r}}=\sum_{s_{1}=0}^{\infty} \ldots \sum_{s_{r}=0}^{\infty} A_{s_{1}, \ldots, s_{r}}
$$

To calculate with this operator MacMahon [15] proposed a list of elimination rules. Here is one of them :

$$
\stackrel{\Omega}{\geq} \frac{1}{(1-\omega x)\left(1-\frac{y}{\omega^{r}}\right)}=\frac{1}{(1-x)\left(1-x^{r} y\right)}, \quad r \geq 0
$$


Our results can be translated in general elimination rules. Let $k$ be a positive integer and $s=\left(s_{1}, \ldots, s_{k}\right)$ be a sequence of integers. Then :

$$
\begin{gathered}
\quad \Omega \frac{1}{1-\omega x} \prod_{i=1}^{k} \frac{1}{1-y_{i} / \omega^{s_{i}}}=\frac{1}{1-x} \prod_{i=1}^{k} \frac{1}{1-x^{s_{i}} y_{i}}, \\
\geq \frac{1}{\geq} \frac{1}{1-x \omega_{1} / \omega_{2}} \prod_{i=1}^{k} \frac{1}{1-y_{i} /\left(\omega_{1} / \omega_{2}\right)^{s_{i}}}=\prod_{i=1}^{k} \frac{1}{1-x^{s_{i}} y_{i}} .
\end{gathered}
$$

\subsection{From Product to Constraint Matrix}

For any sequence $c=\left(c_{1}, \ldots, c_{k}\right)$ of positive integers, can one construct a strictly upper triangular constraint matrix $A[1 . . k, 1 . . k]$ of integers such that $P_{A}$ is a set of compositions with weight generating function $\prod_{i=1}^{k}\left(1-q^{c_{i}}\right)^{-1}$ ? We can answer yes for any sequence. First let us suppose that $c_{1}=1$.

Proposition 1 Given a sequence $\left(c_{1}, \ldots, c_{k}\right)$ of positive integers, such that $c_{1}=1$, there exist

$$
\prod_{i=2}^{k}\left(\begin{array}{c}
c_{i}-1 \\
i-2
\end{array}\right)
$$

upper triangular matrices $B$ with nonnegative integers coefficients and ones on the diagonal such that if $A=I-B^{-1}$ then $P_{A}$ has generating function $\prod_{i=1}^{k}\left(1-q^{c_{i}}\right)^{-1}$.

Proof Given a sequence $\left(c_{1}, \ldots, c_{k}\right)$ of positive integers with $c_{1}=1$, we can always construct an upper triangular matrix $B$ with ones on the diagonal and nonnegative entries such that the sum of the entries in the $j^{t h}$ column is $c_{j}$. Then $B$ is invertible and its inverse is an upper triangular, integer matrix with ones on the diagonal. Thus the matrix $A=I-B^{-1}$ is the constraint matrix $A$ such that $P_{A}$ has generating function $\prod_{i=1}^{k}\left(1-q^{c_{i}}\right)^{-1}$.

Remark If $c_{1}$ is not equal to 1 , we can construct matrices $A[1 . . k+1,1 . . k+1]$ of integers such that $P_{A,}=$ is a set of compositions with weight generating function $\prod_{i=1}^{k}\left(1-q^{c_{i}}\right)^{-1}$.

When the $c_{i}$ satisfy $c_{i} \geq i$, we can use the following corollary to show, in Proposition 2, a simple form for the inequalities defining $P_{A}$.

Corollary 4 If $P_{A}$ is a set of compositions with $\lambda_{i} \geq \lambda_{i+1}$ for $2 \leq i \leq k-1$ and with the first part constrained by $\lambda_{1} \geq \sum_{i=1}^{k-1} a_{i} \lambda_{i+1}$, the generating function of $P_{A}$ is

$$
\frac{1}{1-q} \prod_{i=1}^{k-1} \frac{1}{1-q^{i+a_{1}+\cdots+a_{i}}} .
$$

Proof In this case $B=(I-A)^{-1}$ is the upper triangular matrix defined by $B[1,1]=1$, $B[1, j]=a_{1}+\cdots+a_{j-1}$ for $j>1$, and for $2 \leq i \leq j, B[i, j]=1$. The column sums, $b_{j}$, 
satisfy $b_{1}=1$ and for $2 \leq i \leq k, b_{j}=(j-1)+a_{1}+\cdots+a_{j-1}$, so the result now follows from Theorem 1 .

Proposition 2 For any sequence $c=\left(c_{1}, \ldots, c_{k}\right)$ of positive integers satisfying $c_{i} \geq i-1$, one can construct a matrix $A$ such that either $P_{A}(n, k)$ or $P_{A,=}(n, k+1)$ is a set of compositions with weight generating function $\prod_{i=1}^{k}\left(1-q^{c_{i}}\right)^{-1}$.

Proof If $c_{1}=1$, let $A$ be the $k \times k$ strictly upper triangular matrix such that $A[i, i+1]=1$ if $i \geq 2 ; A[i, j]=0$ if $2 \leq i<j-1 ; A[1,2]=c_{2}-1$; and $A[1, j]=c_{j}-c_{j-1}-1$ for $j>2$. Then by Corollary 4 , using $a_{i}=A[1, i+1], P_{A}(n, k)$ has generating function $\prod_{i=1}^{k}\left(1-q^{b_{i}}\right)^{-1}$, where the $b_{i}$ are defined by $b_{1}=1=c_{1}$ and for $2 \leq i \leq k$

$$
b_{i}=(i-1)+\sum_{j=1}^{i-1} A[1, j+1]=(i-1)+\left(c_{2}-1\right)+\sum_{j=2}^{i-1}\left(c_{j+1}-c_{j}-1\right)=c_{i} .
$$

Note from the proof of Corollary 4 that in this case $B=(I-A)^{-1}$ has all entries nonnegative, since $\sum_{i=1}^{j-1} a_{i}=c_{i}-(i-1) \geq 0$, by the constraints on the $c_{i}$. So, by Lemma $1 P_{A}(n, k)$ is a set of compositions.

If $c_{1}>1$, apply the technique for $c_{1}=1$ to the sequence $c^{\prime}=\left(1, c_{1}, c_{2}, \ldots c_{k}\right)$ to get a family of compositions $P_{A}(n, k)$ with generating function $(1-q)^{-1} \prod_{i=1}^{k}\left(1-q^{c_{i}}\right)^{-1}$. Then $P_{A,=}(n, k)$ is the required set of compositions.

Example 11 Given the sequence $1,3,5, \ldots 2 k-1$, of the first $k$ odd positive integers, the method of Proposition 2 says that $\prod_{i=1}^{k}\left(1-q^{2 i-1}\right)^{-1}$ is the generating function of the partitions $\lambda$ of $n$ with at most $k$ parts and with $\lambda_{1} \geq 2 \lambda_{2}+\sum_{i=3}^{k-1} \lambda_{i}$.

Example 12 Given the sequence $1,4,6,9,11,14, \ldots, 5 k-4,5 k-1$, of the first $2 k$ integers congruent to 1 or $4 \bmod 5$, the method of Proposition 1 says that if we choose $B$ such that

$$
B[i, j]= \begin{cases}0 & \text { if } j<i \\ 1 & \text { if } j=i \\ 3 & \text { if } i \text { odd } \\ 2 & \text { otherwise. }\end{cases}
$$

Then

$$
\prod_{i=1}^{k} \frac{1}{\left(1-q^{5 i-4}\right)\left(1-q^{5 i-1)}\right.}
$$

is the generating function of the partitions $\lambda$ of $n$ with at most $2 k$ parts and with $\lambda_{2 i-1} \geq$ $\sum_{j \geq i} 3\left(2^{j-i}\right)\left(\lambda_{2 j}-\lambda_{2 j+1}\right)$ and $\lambda_{2 i} \geq 2 \lambda_{2 i+1}-\sum_{j>i} 2^{j-i+1}\left(\lambda_{2 j}-\lambda_{2 j+1}\right)$.

Example 13 Given the sequence 2, 2, 4, 4,6,6, .., 2k, 2k, by Proposition 2,

$$
\prod_{i=1}^{k} \frac{1}{\left(1-q^{2 i}\right)^{2}}
$$

is the generating function of the set of compositions satisfying $\lambda_{1} \geq \sum_{i=2}^{2 k}(-1)^{i} \lambda_{i}$ and $\lambda_{i} \geq \lambda_{i+1}$ for $2 \leq i \leq 2 k$. 


\section{Rational Coefficients}

In this section we would like to generalize our results to allow some of the elements of the constraint matrix to be rational. In particular, we will find the generating function for the set of integer sequences $\lambda_{1}, \ldots, \lambda_{k}$ satisfying the constraints:

$$
\begin{aligned}
\lambda_{1} & \geq c_{1}\left\lceil\frac{n_{1}}{d_{1}} \lambda_{2}\right\rceil+\sum_{i=2}^{k} c_{i} \lambda_{i} \\
\lambda_{2} & \geq \frac{n_{2}}{d_{2}} \lambda_{3} \\
\lambda_{3} & \geq \frac{n_{3}}{d_{3}} \lambda_{4} \\
& \vdots \\
\lambda_{k-1} & \geq \frac{n_{k-1}}{d_{k-1}} \lambda_{k} \\
\lambda_{k} & \geq 0
\end{aligned}
$$

where:

- For $1 \leq i \leq k-1, n_{i}$ and $d_{i}$ are positive integers and

- the $c_{i}$ are any integers which make the first constraint strong enough to guarantee that $\lambda_{1} \geq 0$. (We will see several examples of such $c_{i}$.)

For $i=1 \ldots k$, let

$$
a_{i}=\prod_{j=1}^{i-1} d_{j} \prod_{t=i}^{k-1} n_{t} .
$$

Then $a_{i} / a_{i+1}=n_{i} / d_{i}$, so the system (8) above is equivalent to:

$$
\begin{gathered}
\lambda_{1} \geq c_{1}\left\lceil\frac{a_{1}}{a_{2}} \lambda_{2}\right\rceil+\sum_{i=2}^{k} c_{i} \lambda_{i} \text { and } \\
\lambda_{2} / a_{2} \geq \lambda_{3} / a_{3} \geq \cdots \geq \lambda_{k-1} / a_{k-1} \geq \lambda_{k} / a_{k} .
\end{gathered}
$$

\subsection{The Generating Function for (을}

Consider first the case where $c_{1}=1$ and $c_{i}=0$ for $i \geq 2$.

Theorem 2 Given a sequence of positive integers $a_{1}, \ldots, a_{k}$, the generating function for 
the compositions $\lambda_{1}, \ldots, \lambda_{k}$ satisfying

$$
\begin{aligned}
\lambda_{1} & \geq a_{1} \lambda_{2} / a_{2} \\
\lambda_{2} & \geq a_{2} \lambda_{3} / a_{3} \\
& \vdots \\
\lambda_{k-2} & \geq a_{k-2} \lambda_{k-1} / a_{k-1} \\
\lambda_{k-1} & \geq a_{k-1} \lambda_{k} / a_{k} \\
\lambda_{k} & \geq 0
\end{aligned}
$$

is

$$
\frac{\sum_{z_{2}=0}^{a_{2}-1} \sum_{z_{3}=0}^{a_{3}-1} \cdots \sum_{z_{k}=0}^{a_{k}-1} q^{\left\lceil\frac{a_{1} z_{2}}{a_{2}}\right\rceil+\sum_{i=2}^{k} z_{i}} \prod_{i=2}^{k-1} q^{b_{i}\left\lceil\frac{z_{i+1}}{a_{i+1}}-\frac{z_{i}}{a_{i}}\right\rceil}}{\prod_{i=1}^{k}\left(1-q^{b_{i}}\right)}
$$

where $b_{1}=1$ and for $2 \leq i \leq k$,

$$
b_{i}=a_{1}+a_{2}+\cdots+a_{i} .
$$

Proof For $2 \leq i \leq k$, let

$$
\lambda_{i}=a_{i} x_{i}+z_{i}
$$

where $x_{i} \geq 0$ and $0 \leq z_{i}<a_{i}$. The system of inequalities becomes

$$
\begin{aligned}
\lambda_{1} & \geq a_{1} x_{2}+a_{1} z_{2} / a_{2} \\
a_{2} x_{2}+z_{2} & \geq a_{2} x_{3}+a_{2} z_{3} / a_{3} \\
a_{3} x_{3}+z_{3} & \geq a_{3} x_{4}+a_{3} z_{4} / a_{4} \\
& \vdots \\
a_{k-2} x_{k-2}+z_{k-2} & \geq a_{k-2} x_{k-1}+a_{k-2} z_{k-1} / a_{k-1} \\
a_{k-1} x_{k-1}+z_{k-1} & \geq a_{k-1} \lambda_{k} \\
a_{k} x_{k}+z_{k} & \geq 0
\end{aligned}
$$

Rearrange the sequence $\lambda_{1}, \lambda_{2}, \ldots, \lambda_{k}$ by moving $\left(a_{i}-1\right) x_{i}+z_{i}$ "dots" in the Ferrers diagram from part $\lambda_{i}$ to part $\lambda_{1}$ for $2 \leq i \leq k$ to get a new sequence of the same weight:

$$
x_{1}, x_{2}, \ldots, x_{k}
$$

where

$$
x_{1}=\lambda_{1}+\sum_{i=2}^{k}\left(\left(a_{i}-1\right) x_{i}+z_{i}\right)
$$


satisfying:

$$
\begin{aligned}
x_{1} & \geq\left(a_{1}+a_{2}-1\right) x_{2}+\sum_{i=3}^{k}\left(a_{i}-1\right) x_{i}+a_{1} z_{2} / a_{2}+\sum_{i=2}^{k} z_{i} \\
x_{2} & \geq x_{3}+z_{3} / a_{3}-z_{2} / a_{2} \\
x_{3} & \geq x_{4}+z_{4} / a_{4}-z_{3} / a_{3} \\
& \vdots \\
x_{k-2} & \geq x_{k-1}+z_{k-1} / a_{k-1}-z_{k-2} / a_{k-2} \\
x_{k-1} & \geq x_{k}+z_{k} / a_{k}-z_{k-1} / a_{k-1} \\
x_{k} & \geq 0 .
\end{aligned}
$$

This is just the system

$$
\begin{aligned}
& x_{1} \geq\left(a_{1}+a_{2}-1\right) x_{2}+\sum_{i=3}^{k}\left(a_{i}-1\right) x_{i}+s_{1} \\
& x_{i} \geq x_{i+1}+s_{i} \quad 2 \leq i \leq k-1 \\
& x_{k} \geq 0
\end{aligned}
$$

where

$$
s_{1}=\left\lceil a_{1} z_{2} / a_{2}\right\rceil+\sum_{i=2}^{k} z_{i}
$$

and

$$
s_{i}=\left\lceil z_{i+1} / a_{i+1}-z_{i} / a_{i}\right\rceil
$$

for $2 \leq i \leq k$.

Thus, by Corollary 3 and Corollary 4 , the generating function for fixed $s_{1}, \ldots s_{k-1}$ is

$$
\frac{\prod_{i=1}^{k-1} q^{b_{i} s_{i}}}{\prod_{i=1}^{k}\left(1-q^{b_{i}}\right)},
$$

where $b_{1}=1$ and for $2 \leq i \leq k, b_{i}=a_{1}+a_{2}+\cdots+a_{i}$. Summing over all possible sequences $s_{1}, s_{2}, \ldots, s_{k-1}$ as the $z_{i}$ vary independently from 0 to $a_{i}-1$ gives the result.

Example 14 Suppose $\left(a_{1}, a_{2}, a_{3}, a_{4}\right)=(4,3,2,1)$. Then $\left(b_{1}, b_{2}, b_{3}, b_{4}\right)=(1,7,9,10)$ so the denominator of (9) is the product $(1-q)\left(1-q^{7}\right)\left(1-q^{9}\right)\left(1-q^{10}\right)$. The numerator of (9) is the sum, as $\left(z_{2}, z_{3}, z_{4}\right)$ range over the set $\{(0,0,0),(1,0,0),(2,0,0),(0,1,0),(1,1,0),(2,1,0)\}$, of the terms

$$
q^{\left\lceil 4 z_{2} / 3\right\rceil+z_{2}+z_{3}+z_{4}} q^{7\left\lceil z_{3} / 2-z_{2} / 3\right\rceil} q^{9\left\lceil z_{4} / 1-z_{3} / 2\right\rceil}=q^{\left\lceil 4 z_{2} / 3\right\rceil+z_{2}+z_{3}} q^{7\left\lceil z_{3} / 2-z_{2} / 3\right\rceil} .
$$

So, the generating function is

$$
\begin{aligned}
\frac{1+q^{3}+q^{5}+q^{8}+q^{11}+q^{6}}{(1-q)\left(1-q^{7}\right)\left(1-q^{9}\right)\left(1-q^{10}\right)} & =\frac{\left(1+q^{3}+q^{6}\right)\left(1+q^{5}\right)}{(1-q)\left(1-q^{7}\right)\left(1-q^{9}\right)\left(1-q^{10}\right)} \\
& =\frac{1}{(1-q)\left(1-q^{7}\right)\left(1-q^{3}\right)\left(1-q^{5}\right)}
\end{aligned}
$$


More generally, in the special case of Theorem 2 that $a_{i}=k-i+1$ for $1 \leq i \leq k$ and where $c_{1}=1$ and $c_{i}=0$ for $1=2, \ldots k$, the constraints become:

$$
\lambda_{1} / k \geq \lambda_{2} /(k-1) \geq \cdots \geq \lambda_{k-1} / 2 \geq \lambda_{k} / 1
$$

and the sequences satisfying these constraints are partitions known as lecture hall partitions. It was first shown in [10] that these partitions have generating function

$$
\prod_{i=1}^{k} \frac{1}{\left(1-q^{2 i-1}\right)}
$$

giving a finite form of the identity of Euler equating partitions of $n$ into distinct parts with partitions of $n$ into odd parts.

Note that although Theorem 2 gives an explicit form of the generating function for these partitions, it does not help with the factoring of the numerator. In another proof of the lecture hall partitions theorem [1], Andrews uses partition analysis to get the generating function and shows how to factor the numerator via a permutation of the set of tuples $\left(z_{2}, \ldots z_{k}\right)$.

Example 15 Suppose $\left(a_{1}, a_{2}, a_{3}, a_{4}, a_{5}\right)=(1,3,2,3,1)$. Then $\left(b_{1}, b_{2}, b_{3}, b_{4}, b_{5}\right)=(1,4,6,9,10)$ so the denominator of (9) is the product $(1-q)\left(1-q^{4}\right)\left(1-q^{6}\right)\left(1-q^{9}\right)\left(1-q^{10}\right)$. The numerator of (9) is the sum, as $\left(z_{2}, z_{3}, z_{4}, z_{5}\right)$ ranges over the set $\{(0,0,0,0),(1,0,0,0),(2,0,0,0)$, $(0,1,0,0),(1,1,0,0),(2,1,0,0),(0,0,1,0),(1,0,1,0),(2,0,1,0),(0,1,1,0),(1,1,1,0),(2,1,1,0)$, $(0,0,2,0),(1,0,2,0),(2,0,2,0),(0,1,2,0),(1,1,2,0),(2,1,2,0)\}$, of the terms

$$
q^{\left\lceil z_{2} / 3\right\rceil+z_{2}+z_{3}+z_{4}} q^{4\left\lceil z_{3} / 2-z_{2} / 3\right\rceil} q^{6\left\lceil z_{4} / 3-z_{3} / 2\right\rceil} q^{9\left\lceil z_{5} / 1-z_{4} / 3\right\rceil} .
$$

So, the generating function is

$$
\begin{array}{r}
\frac{1+q^{2}+q^{3}+q^{5}+q^{7}+q^{4}+q^{7}+q^{9}+q^{10}+q^{6}+q^{8}+q^{5}+q^{8}+q^{10}+q^{11}+q^{13}+q^{15}+q^{12}}{(1-q)\left(1-q^{4}\right)\left(1-q^{6}\right)\left(1-q^{9}\right)\left(1-q^{10}\right)} \\
=\frac{\left(1+q^{2}+q^{4}\right)\left(1+q^{3}+q^{6}\right)\left(1+q^{5}\right)}{(1-q)\left(1-q^{4}\right)\left(1-q^{6}\right)\left(1-q^{9}\right)\left(1-q^{10}\right)} \\
=\frac{1}{(1-q)\left(1-q^{2}\right)\left(1-q^{3}\right)\left(1-q^{4}\right)\left(1-q^{5}\right)} .
\end{array}
$$

Thus there is a one-to-one correspondence between the compositions of $n$ into 5 parts satisfying

$$
\frac{\lambda_{1}}{1} \geq \frac{\lambda_{2}}{3} \geq \frac{\lambda_{3}}{2} \geq \frac{\lambda_{4}}{3} \geq \frac{\lambda_{5}}{1}
$$

and the partitions of $n$ into parts of size at most 5 .

It is straightforward to extend Theorem 2 to get the generating function for the system (8) with more general $c_{i}$.

Corollary 5 Given a sequence of positive integers $a_{1}, \ldots, a_{k}$ and a sequence of integers $c_{1}, \ldots, c_{k}$, consider the set of sequences $\lambda_{1}, \ldots, \lambda_{k}$ satisfying

$$
\lambda_{1} \geq c_{1}\left\lceil\frac{a_{1}}{a_{2}} \lambda_{2}\right\rceil+\sum_{i=2}^{k} c_{i} \lambda_{i} \quad \text { and }
$$




$$
\lambda_{2} / a_{2} \geq \lambda_{3} / a_{3} \geq \cdots \geq \lambda_{k-1} / a_{k-1} \geq \lambda_{k} / a_{k}
$$

As long as the $c_{i}$ are integers which make the first constraint strong enough to guarantee that $\lambda_{1} \geq 0$, the generating function is

$$
\frac{\sum_{z_{2}=0}^{a_{2}-1} \sum_{z_{3}=0}^{a_{3}-1} \cdots \sum_{z_{k}=0}^{a_{k}-1} q^{c_{1}\left\lceil\frac{a_{1} z_{2}}{a_{2}}\right\rceil+\sum_{i=2}^{k}\left(c_{i}+1\right) z_{i}} \prod_{i=2}^{k-1} q^{b_{i}\left\lceil\frac{z_{i+1}}{a_{i+1}}-\frac{z_{i}}{a_{i}}\right\rceil}}{\prod_{i=1}^{k}\left(1-q^{b_{i}}\right)},
$$

where $b_{1}=1, b_{2}=c_{1} a_{1}$ and for $3 \leq i \leq k$,

$$
b_{i}=c_{1} a_{1}+\left(c_{2}+1\right) a_{2}+\cdots+\left(c_{i}+1\right) a_{i} .
$$

Proof Using the same strategy as in the proof of Theorem 2, the system of inequalities in the Corollary becomes

$$
\begin{aligned}
& x_{1} \geq\left(c_{1} a_{1}+\left(c_{2}+1\right) a_{2}-1\right) x_{2}+\sum_{i=3}^{k}\left(\left(c_{i}+1\right) a_{i}-1\right) x_{i}+s_{1} \\
& x_{i} \geq x_{i+1}+s_{i} \quad 2 \leq i \leq k-1 \\
& x_{k} \geq 0
\end{aligned}
$$

where now $0 \leq z_{i} \leq i-1$,

$$
s_{1}=c_{1}\left\lceil a_{1} z_{2} / a_{2}\right\rceil+\sum_{i=2}^{k}\left(c_{i}+1\right) z_{i}
$$

and

$$
s_{i}=\left\lceil z_{i+1} / a_{i+1}-z_{i} / a_{i}\right\rceil
$$

for $2 \leq i \leq k-1$. Thus, by Corollary 3 and Corollary 4 , the generating function for fixed $s_{1}, s_{2}, \ldots s_{k-1}$ is

$$
\frac{\prod_{i=1}^{k-1} q^{b_{i} s_{i}}}{\prod_{i=1}^{k}\left(1-q^{b_{i}}\right)}
$$

where $b_{1}=1, b_{2}=c_{1} a_{1}$ and for $3 \leq i \leq k, b_{i}=c_{1} a_{1}+\left(c_{2}+1\right) a_{2}+\left(c_{3}+1\right) a_{3}+\cdots+\left(c_{i}+1\right) a_{i}$. Summing over all possible sequences $s_{1}, s_{2}, \ldots s_{k-1}$ as the $z_{i}$ vary independently from 0 to $a_{i}-1$ gives the result.

Example 16 Consider sequences $\left(\lambda_{1}, \lambda_{2}, \lambda_{3}, \lambda_{4}, \lambda_{5}\right)$ satisfying

$$
\lambda_{1} \geq \lambda_{2} / 2+3 \lambda_{5}
$$

and

$$
\frac{\lambda_{2}}{2} \geq \frac{\lambda_{3}}{1} \geq \frac{\lambda_{4}}{2} \geq \frac{\lambda_{5}}{1} \geq 0
$$

The constraints guarantee these $\lambda$ are compositions and they satisfy the conditions of Corollary 5 with $k=5,\left(a_{1}, a_{2}, a_{3}, a_{4}, a_{5}\right)=(1,2,1,2,1)$ and $\left(c_{1}, c_{2}, c_{3}, c_{4}, c_{5}\right)=(1,0,0,0,3)$. 
Then $\left(b_{1}, b_{2}, b_{3}, b_{4}, b_{5}\right)=(1,3,4,6,10)$ so the denominator of (11) is the product $(1-q)(1-$ $\left.q^{3}\right)\left(1-q^{4}\right)\left(1-q^{6}\right)\left(1-q^{10}\right)$. The numerator of (11) is the sum of the terms

$$
q^{\left\lceil z_{2} / 2\right\rceil+z_{2}+z_{3}+z_{4}+4 z_{4}} q^{3\left\lceil z_{3} / 1-z_{2} / 2\right\rceil} q^{4\left\lceil z_{4} / 2-z_{3} / 1\right\rceil}
$$

as $\left(z_{2}, z_{3}, z_{4}, z_{5}\right)$ ranges over the set $\{(0,0,0,0),(1,0,0,0),(0,0,1,0),(1,0,1,0)$,$\} . So, by$ Corollary 5 , the generating function is

$$
\begin{aligned}
\frac{1+q^{2}+q^{5}+q^{7}}{(1-q)\left(1-q^{3}\right)\left(1-q^{4}\right)\left(1-q^{6}\right)\left(1-q^{10}\right)} & =\frac{\left(1+q^{2}\right)\left(1+q^{5}\right)}{(1-q)\left(1-q^{3}\right)\left(1-q^{4}\right)\left(1-q^{6}\right)\left(1-q^{10}\right)} \\
& =\frac{1}{(1-q)\left(1-q^{2}\right)\left(1-q^{3}\right)\left(1-q^{5}\right)\left(1-q^{6}\right)} .
\end{aligned}
$$

Example 17 Consider sequences $\left(\lambda_{1}, \lambda_{2}, \lambda_{3}, \lambda_{4}\right)$ satisfying

$$
\lambda_{1} \geq 2\left\lceil 7 \lambda_{2} / 3\right\rceil+3 \lambda_{2}+\lambda_{3}+5 \lambda_{4}
$$

and

$$
\frac{\lambda_{2}}{3} \geq \frac{\lambda_{3}}{2} \geq \frac{\lambda_{4}}{1} \geq 0
$$

The constraints guarantee these $\lambda$ are partitions and they satisfy the conditions of Corollary 5 with $k=4,\left(a_{1}, a_{2}, a_{3}, a_{4}\right)=(7,3,2,1)$ and $\left(c_{1}, c_{2}, c_{3}, c_{4}\right)=(2,3,1,5)$. Then $\left(b_{1}, b_{2}, b_{3}, b_{4}\right)=(1,26,30,36)$ so the denominator of (11) is the product $(1-q)\left(1-q^{26}\right)(1-$ $\left.q^{30}\right)\left(1-q^{36}\right)$. The numerator of (11) is the sum of the terms

$$
q^{2\left\lceil 7 z_{2} / 3\right\rceil+4 z_{2}+2 z_{3}+6 z_{4}} q^{26\left\lceil z_{3} / 2-z_{2} / 3\right\rceil} q^{30\left\lceil z_{4} / 1-z_{3} / 2\right\rceil}
$$

as $\left(z_{2}, z_{3}, z_{4}\right)$ ranges over the set $\{(0,0,0),(1,0,0),(2,0,0),(0,1,0),(1,1,0),(2,1,0)\}$. So, by Corollary 5 , the generating function is

$$
\begin{aligned}
\frac{1+q^{10}+q^{18}+q^{28}+q^{38}+q^{20}}{(1-q)\left(1-q^{26}\right)\left(1-q^{30}\right)\left(1-q^{36}\right)} & =\frac{\left(1+q^{10}+q^{20}\right)\left(1+q^{18}\right)}{(1-q)\left(1-q^{26}\right)\left(1-q^{30}\right)\left(1-q^{36}\right)} \\
& =\frac{1}{(1-q)\left(1-q^{10}\right)\left(1-q^{18}\right)\left(1-q^{26}\right)}
\end{aligned}
$$

\section{$3.2 \quad$ A Special Case}

Corollary 6 Suppose the sequence $a_{1}, a_{2}, \ldots, a_{k}$ has the property that for $1 \leq i \leq k-1$ if $a_{i}>1$, then $a_{i+1}=1$. Then there is a one-to-one correspondence between the compositions $\lambda_{1}, \ldots, \lambda_{k}$ of $n$ satisfying

$$
\lambda_{1} / a_{1} \geq \lambda_{2} / a_{2} \geq \lambda_{3} / a_{3} \geq \cdots \geq \lambda_{k-1} / a_{k-1} \geq \lambda_{k} / a_{k}
$$

and the partitions of $n$ into parts in

$$
\left\{1, b_{2}, b_{2}+1, \ldots, b_{k}\right\},
$$

where $b_{1}=1, b_{2}=a_{1}+a_{2}$ and $b_{i+1}=b_{i}+a_{i+1}$ for $2 \leq i \leq k-1$, such that at most one part can appear from each of the sets

$$
S_{i}=\left\{p \mid b_{i}+1 \leq p \leq b_{i+1}-1\right\} .
$$


Proof Since $z_{j}=0$ whenever $a_{j}=1$, the generating function (91) becomes

$$
\frac{\sum_{z_{2}=0}^{a_{2}-1} \sum_{z_{3}=0}^{a_{3}-1} \cdots \sum_{z_{k}=0}^{a_{k}-1} q^{\left\lceil\frac{a_{1} z_{2}}{a_{2}}\right\rceil+z_{2}} \prod_{i=2: a_{i+1}>1}^{k-1} q^{b_{i}\left\lceil\frac{z_{i+1}}{a_{i+1}}\right\rceil+z_{i+1}}}{\prod_{i=1}^{k}\left(1-q^{b_{i}}\right)}
$$

and since consecutive $a_{i}$ cannot both be greater than 1 , we get

$$
\frac{\sum_{z_{2}=0}^{a_{2}-1} q^{\left\lceil\frac{a_{1} z_{2}}{a_{2}}\right\rceil+z_{2}} \prod_{i=2: a_{i+1}>1}^{k-1} \sum_{z_{i+1}=0}^{a_{i+1}-1} q^{b_{i}\left\lceil\frac{z_{i+1}}{a_{i+1}}\right\rceil+z_{i+1}}}{\prod_{i=1}^{k}\left(1-q^{b_{i}}\right)}
$$

which, letting $b_{1}=1$, gives

$$
\frac{\prod_{i=1: a_{i+1}>1}^{k-1} \sum_{z_{i+1}=0}^{a_{i+1}-1} q^{b_{i}\left\lceil\frac{z_{i+1}}{a_{i+1}}\right\rceil+z_{i+1}}}{\prod_{i=1}^{k}\left(1-q^{b_{i}}\right)}
$$

Since $b_{2}=a_{1}+a_{2}$ and $b_{i+1}=b_{i}+a_{i+1}$ we get

$$
\frac{\prod_{i=1: b_{i+1}-b_{i}>1}^{k-2}\left(1+q^{b_{i}+1}+q^{b_{i}+2}+\cdots+q^{b_{i+1}-1}\right)}{\prod_{i=1}^{k}\left(1-q^{b_{i}}\right)} .
$$

Note that each integer $m$ satisfying $b_{2} \leq m \leq b_{k}$ appears exactly once as an exponent in either the numerator or denominator. So (12) is the generating function for partitions into parts in

$$
\left\{1, b_{2}, b_{2}+1, \ldots, b_{k}\right\}
$$

such that at most one part can appear from each of the sets

$$
S_{i}=\left\{p \mid b_{i}+1 \leq p \leq b_{i+1}-1\right\} .
$$

(The set $S_{i}$ is empty if $b_{i+1}-b_{i}>1$ ). This result can easily be proved bijectively.

Example 18 Compositions of $n$ satisfying

$$
\lambda_{1} \geq \lambda_{2} \geq \frac{\lambda_{3}}{2} \geq \lambda_{4} \geq \frac{\lambda_{5}}{2} \geq \lambda_{6} \geq \cdots \geq \lambda_{2 k} \geq \frac{\lambda_{2 k+1}}{2} \geq 0
$$

are in one-to-one correspondence with the set of partitions of $n$ into parts of size at most $3 k+2$ in which parts divisible by 3 can appear at most once.

Example 19 Compositions of $n$ satisfying

$$
\lambda_{1} \geq \frac{\lambda_{2}}{2} \geq \lambda_{3} \geq \frac{\lambda_{4}}{3} \geq \lambda_{5} \geq \frac{\lambda_{6}}{4} \geq \cdots \geq \lambda_{2 k-1} \geq \frac{\lambda_{2 k}}{k+1}
$$

are in one-to-one correspondence with the set of partitions of $n$ into parts of size at most $k(k+5) / 2$, such that for each $j \geq 3$, at most one part can occur from the set

$$
\left\{i \mid\left(\begin{array}{l}
j \\
2
\end{array}\right) \leq i+1 \leq\left(\begin{array}{l}
j \\
2
\end{array}\right)+(j-3)\right\} \text {. }
$$




\section{Two Variable Generating Functions}

In their study of lecture hall partitions, Bousquet-Mélou and Eriksson found it very useful to consider the 2-variable (odd/even weighted) generating function of the set of partitions satisfying the lecture hall constraints. We show here how our method can be adapted to get multivariable generating functions for compositions satisfying linear constraints, using the two-variable case as an example.

Given a sequence $\lambda=\left(\lambda_{1}, \ldots, \lambda_{k}\right)$, we denote by $\lambda_{o}$ the subsequence $\left(\lambda_{1}, \lambda_{3}, \lambda_{5}, \ldots\right)$ and by $\lambda_{e}$ the subsequence $\left(\lambda_{2}, \lambda_{4}, \lambda_{6}, \ldots\right)$.

\subsection{Integer Coefficients}

Let $A[1 . . k, 1 . . k]$ be a strictly upper triangular matrix of integers, such that $P_{A}$ is a set of compositions. Let $Q_{A}(l, m, k)$ be the subset of $P_{A}$ consisting of those compositions $\lambda=\left(\lambda_{1}, \lambda_{2}, \cdots, \lambda_{k}\right)$, with $l=\left|\lambda_{o}\right|=\lambda_{1}+\lambda_{3}+\lambda_{5}+\cdots$ and with $m=\left|\lambda_{e}\right|=\lambda_{2}+\lambda_{4}+\lambda_{6}+\cdots$, satisfying

$$
\lambda_{i} \geq \sum_{j=i+1}^{k} A[i, j] \lambda_{j} \quad \text { for } i \geq 1 .
$$

We would now like to write the generating function

$$
\sum_{l, m \geq 0}\left|Q_{A}(l, m, k)\right| x^{l} y^{m}
$$

Theorem 3 Let $A$ a $k \times k$ strictly upper triangular matrix of integers,. If $B=I+A+$ $A^{2}+\ldots+A^{k-1}$ has nonnegative coefficients then the two-variable generating function for $Q_{A}(l, m, k)$ is

$$
\prod_{i=1}^{k} \frac{1}{1-x^{o_{i}} y^{e_{i}}}
$$

where $o_{i}=\sum_{j \geq 1} B[2 j-1, i]$ and $e_{i}=\sum_{j \geq 1} B[2 j, i]$.

Proof As $\lambda=B\left[s_{1}, \ldots, s_{k}\right]^{T}$ then $\lambda_{2 i+1}=\sum_{j} B[2 j+1, j] s_{j}$ and $\lambda_{2 i}=\sum_{j} B[2 j, i] s_{j}$. Therefore $\left|\lambda_{o}\right|=\sum_{i \geq 1} o_{i} s_{i}$ and $\left|\lambda_{e}\right|=\sum_{i \geq 1} e_{i} s_{i}$. The result follows.

Example 20 Let $f_{k}(x, y)$ be the generating function for ordinary partitions:

$$
\lambda_{1} \geq \lambda_{2} \geq \cdots \geq \lambda_{k} \geq 0 .
$$

Then $o_{1}, o_{2}, o_{3}, \ldots$ is: $1,1,2,2,3,3,4,4, \ldots$ and $e_{1}, e_{2}, e_{3}, \ldots$ is: $0,1,1,2,2,3,3,4,4, \ldots$ so the generating function is

$$
f_{k}(x, y)=\prod_{i=1}^{\lceil k / 2\rceil} \frac{1}{1-x^{i} y^{i-1}} \prod_{i=1}^{\lfloor k / 2\rfloor} \frac{1}{1-x^{i} y^{i}}
$$


Note that

$$
f_{k+1}(x, y)=\frac{f_{k}\left(x^{2} y, x^{-1}\right)}{(1-x)}
$$

\subsection{Alpha-beta Sequences}

Let $A$ be a $k \times k$ strictly upper triangular matrix of integers, such that $P_{A}$ is a set of partitions and let $G(x, y)$ be the two variable generating function, that is,

$$
G(x, y)=\sum_{l, m \geq 0}\left|Q_{A}(l, m)\right| x^{l} y^{m} .
$$

Theorem 4 Let $\alpha \geq 1$ and $\beta \leq \alpha$ be integers (note $\beta$ can be negative). The set of compositions $\lambda_{0}, \lambda_{1}, \ldots \lambda_{k}$, satisfying

$$
\lambda_{0}=\alpha\left(\lambda_{1}+\lambda_{3}+\lambda_{5}+\cdots\right)-\beta\left(\lambda_{2}+\lambda_{4}+\lambda_{6}+\cdots\right)
$$

and for $1 \leq i \leq k, \lambda_{i} \geq \sum_{j=i+1}^{k} A[i, j] \lambda_{j}$ has generating function $G\left(x^{\alpha} y, x^{-\beta+1}\right)$, where $G$ is defined by (14).

Proof Let partition $\lambda=\left(\lambda_{1}, \lambda_{2}, \ldots \lambda_{k}\right)$, satisfy the constraints $\lambda_{i} \geq \sum_{j=i+1}^{k} A[i, j] \lambda_{j}$. Prepend part $\lambda_{0}$ to satisfy the new constraint and call the new composition $\lambda^{\prime}$. (Since $\lambda$ is a partition, the conditions on $\alpha, \beta$ ensure $\lambda_{0} \geq 0$. The odd parts of $\lambda$ become the even parts of $\lambda^{\prime}$. The odd parts of $\lambda^{\prime}$ are the even parts of $\lambda$ plus the new part $\lambda_{0}$, so

$$
\left|\lambda^{\prime}\right|_{e}=|\lambda|_{o} ; \quad\left|\lambda^{\prime}\right|_{o}=|\lambda|_{e}+\alpha|\lambda|_{o}-\beta|\lambda|_{e}
$$

Corollary 7 For fixed constants $\alpha \geq 1$ and $\beta \leq \alpha$, the sequences $\left(\lambda_{1}, \ldots \lambda_{k}\right)$ satisfying, for $1 \leq i \leq k$,

$$
\lambda_{i} \geq \alpha\left(\lambda_{i+1}+\lambda_{i+3}+\lambda_{i+5}+\cdots\right)-\beta\left(\lambda_{i+2}+\lambda_{i+4}+\lambda_{i+6}+\cdots\right),
$$

form a set of compositions with odd/even generating function

$$
G_{k}(x, y)=\prod_{i=1}^{k} \frac{1}{\left(1-x^{o_{i}} y^{e_{i}}\right)}
$$

where

$$
o_{1}=1 ; \quad o_{2}=\alpha ; \quad o_{i}=\alpha o_{i-1}+(-\beta+1) o_{i-2} \quad \text { for } i>2
$$

and

$$
e_{1}=0 ; \quad e_{2}=1 ; \quad e_{i}=o_{i-1} \text { for } i>2 \text {. }
$$


Proof The constraints ensure that $\lambda_{i} \geq 0$ for $i=1, \ldots k$, so as in proof of Theorem 4 their generating functions satisfy the recurrence

$$
G_{k+1}(x, y)=G_{k}\left(x^{\alpha} y, x^{-\beta+1}\right) /(1-x) .
$$

Example 21 If $\alpha=1$ and $\beta=-1$ in Corollary $\mathbf{7}$ we get:

$$
o_{1}=1 ; \quad o_{2}=1 ; \quad o_{i}=o_{i-1}+2 o_{i-2}
$$

and

$$
e_{0}=1 ; \quad e_{1}=2 ; \quad e_{i}=o_{i-1}
$$

so the generating function is

$$
\frac{1}{(1-x)(1-x y)\left(1-x^{3} y\right)\left(1-x^{5} y^{3}\right)\left(1-x^{11} y^{5}\right)\left(1-x^{21} y^{11}\right) \cdots} .
$$

Substituting $x=y=q$ tells us that the partitions $\lambda_{1}, \ldots, \lambda_{k}$ of $n$ satisfying

$$
\lambda_{i} \geq \lambda_{i+1}+\lambda_{i+2}+\lambda_{i+3}+\cdots
$$

are in one-to-one correspondence with partitions of $n$ whose parts must be one of the first $n$ powers of $2: 1,2, \ldots, 2^{n-1}$.

Example 22 In Corollary 17, if $\alpha=1$ and $\beta=1$, we get:

$$
o_{1}=1 ; \quad o_{2}=1 ; \quad o_{i}=o_{i-1}
$$

and

$$
e_{1}=0 ; \quad e_{2}=1 ; \quad e_{i}=o_{i-1},
$$

so the generating function is

$$
\frac{1}{(1-x)(1-x y)(1-x y)(1-x y)(1-x y)(1-x y) \cdots} \text {. }
$$

Substituting $x=y=q$ tells us that the compositions $\lambda_{1}, \ldots, \lambda_{k}$ of $n$ satisfying

$$
\lambda_{i} \geq \sum_{j=1}^{k-i}(-1)^{j} \lambda_{i+j}
$$

are in one-to-one correspondence with compositions of $n$ into $k$ parts whose first part can be any nonnegative integer but whose other parts must be nonnegative even integers. (Compare with Example 2.)

Example 23 If $\alpha=2$ and $\beta=2$, from Corollary 7 we get:

$$
o_{1}=1 ; \quad o_{2}=2 ; \quad o_{i}=2 o_{i-1}-o_{i-2}
$$

and

$$
e_{1}=0 ; \quad e_{2}=1 ; \quad e_{i}=o_{i-1}
$$


so the generating function is

$$
\frac{1}{(1-x)\left(1-x^{2} y\right)\left(1-x^{3} y^{2}\right)\left(1-x^{4} y^{3}\right)\left(1-x^{5} y^{4}\right)\left(1-x^{6} y^{5}\right) \cdots} .
$$

Substituting $x=y=q$ tells us that the partitions $\lambda_{1}, \ldots, \lambda_{k}$ of $n$ satisfying

$$
\lambda_{i} \geq 2 \sum_{j=1}^{k-i}(-1)^{j} \lambda_{i+j}
$$

are in one-to-one correspondence with partitions into odd parts less than $2 k$.

\subsection{The Two Variable Generating Function for Rational Diagonal Con- straints}

To get the odd/even weighted generating function for the compositions satisfying the constraints:

$$
\frac{\lambda_{1}}{a_{1}} \geq \frac{\lambda_{2}}{a_{2}} \geq \frac{\lambda_{3}}{a_{3}} \geq \cdots \geq \frac{\lambda_{k-1}}{a_{k-1}} \geq \frac{\lambda_{k}}{a_{k}} \geq 0,
$$

in the system (10) we separate the "dots" comprising $x_{1}$ into those which come from odd parts:

$$
a_{1} x_{2}+\left(a_{3}-1\right) x_{3}+\left(a_{5}-1\right) x_{5}+\cdots+z_{3}+z_{5}+\cdots
$$

and those which come from even parts:

$$
\left(a_{2}-1\right) x_{2}+\left(a_{4}-1\right) x_{4}+\cdots+z_{2}+z_{4}+z_{6}+\cdots
$$

Then we get the following refinement of (9):

$$
G(x, y)=\frac{\sum_{z_{2}=0}^{a_{2}-1} \cdots \sum_{z_{k}=0}^{a_{k}-1} x^{\left\lceil\frac{a_{1} z_{2}}{a_{2}}\right\rceil+z_{3}+z_{5}+\cdots} y^{z_{2}+z_{4}+\cdots} \prod_{i=2}^{k-1}\left(x^{o_{i}} y^{e_{i}}\right)^{\left\lceil\frac{z_{i+1}}{a_{i+1}}-\frac{z_{i}}{a_{i}}\right\rceil}}{\prod_{i=1}^{k}\left(1-x^{o_{i}} y^{e_{i}}\right)}
$$

where $o_{1}=1, e_{1}=0$, and for $2 \leq i \leq k o_{i}=a_{1}+a_{3}+a_{5}+\cdots+a_{2\lfloor(i-2) / 2\rfloor+1}$ and $e_{i}=a_{2}+a_{4}+a_{6}+\cdots+a_{2\lceil(i-1) / 2\rceil}$.

\subsection{Variations on Lecture Hall Partitions}

Theorem 5 If $G(x, y)=H(x, y) /(1-x)$ is the generating function given in (16)), then whenever $a_{1} \geq a_{2} \geq \cdots \geq a_{k}$, for any $l>0$ and $j \geq 2-l, H\left(x^{l}, x^{j-1} y\right)$ is the generating function for the partitions satisfying

$$
\begin{aligned}
& \lambda_{1}=l\left\lceil a_{1} \lambda_{2} / a_{2}\right\rceil+\sum_{i \geq 1}\left((j-1) \lambda_{2 i}+(l-1) \lambda_{2 i+1}\right) \\
& \frac{\lambda_{2}}{a_{2}} \geq \frac{\lambda_{3}}{a_{3}} \geq \cdots \geq \frac{\lambda_{k-1}}{a_{k-1}} \geq \frac{\lambda_{k}}{a_{k}} \geq 0 .
\end{aligned}
$$


Proof If $j=l=1$, the system (17) is the same as (15), except with equality for $\lambda_{1}$, and the generating function is $H(x, y)=G(x, y) /(1-x)$. Suppose $\lambda$ satisfies (15). To transform $\lambda$ into a composition satisfying (17), we increase the first part by $(l-1)|\lambda|_{o}+(j-1)|\lambda|_{e}$ to get $\lambda^{\prime}$. The conditions of the theorem guarantee that this increase is nonnegative. Then $\left|\lambda^{\prime}\right|_{e}=|\lambda|_{e}$ and

$$
\left|\lambda^{\prime}\right|_{o}=|\lambda|_{o}+(l-1)|\lambda|_{o}+(j-1)|\lambda|_{e}=l|\lambda|_{o}+(j-1)|\lambda|_{e}
$$

Remark If the $a_{i}$ s are not non-increasing then the theorem is still true if $l$ and $j$ are both positive.

We can use Theorem 5 to generalize the Lecture Hall Partition theorem of BousquetMélou and Eriksson.

Corollary 8 For $l>0$ and $j \geq 2-l$, the generating function for the sequences $\lambda_{1}, \ldots, \lambda_{k}$ satisfying

$$
\lambda_{1} \geq l\left\lceil k \lambda_{2} /(k-1)\right\rceil+(j-1)\left(\lambda_{2}+\lambda_{4}+\lambda_{6}+\cdots\right)+(l-1)\left(\lambda_{3}+\lambda_{5}+\lambda_{7}+\cdots\right)
$$

and

$$
\frac{\lambda_{2}}{k-1} \geq \frac{\lambda_{3}}{k-2} \geq \cdots \geq \frac{\lambda_{k-1}}{2} \geq \frac{\lambda_{k}}{1} \geq 0
$$

$i s$

$$
\frac{1}{(1-q)} \prod_{i=1}^{k-1} \frac{1}{\left(1-q^{i l+i j+l}\right)}
$$

Proof Bousquet-Mélou and Eriksson have shown in [10] that when $j=l=1$ the generating function is:

$$
G(x, y)=\prod_{i=0}^{k-1} \frac{1}{\left(1-x^{i+1} y^{i}\right)} .
$$

The result follows from Theorem 5 setting $x=y=q$.

\section{Example 24}

$$
\prod_{i=1}^{k-1} \frac{1}{\left(1-q^{2 i+3}\right)}
$$

is the generating function for the partitions satisfying

$$
\lambda_{1}=3\left\lceil k \lambda_{2} /(k-1)\right\rceil+2 \sum_{j=1}^{k-1}(-1)^{j} \lambda_{1+j}
$$

and

$$
\frac{\lambda_{2}}{k-1} \geq \frac{\lambda_{3}}{k-2} \geq \cdots \geq \frac{\lambda_{k-1}}{2} \geq \frac{\lambda_{k}}{1} .
$$

This is Corollary 8 with $l=3$ and $j=-1$. 


\section{Conclusion}

In this paper we have shown that compositions and partitions defined by (in)equalities have nice and easy-to-compute generating functions in the integer case and, sometimes, in the rational case. The proofs are all bijective and the techniques are powerful and well suited. However these techniques are not completely satisfactory for analyzing families like the Lecture Hall partitions, since additional (and non-trivial) methods may be required to compute a factorization of the numerator of the resulting generating function.

We have found the techniques in this paper to be most useful when implemented and used in conjunction with a computer algebra package like Maple. Through computer experiments, we have been able to discover new identities, whose proof is beyond the scope of these methods. However, we show in a forthcoming article [13] that methods inspired from Bousquet-Mélou and Eriksson [1] and Aa Ya Yee [17, 18] can give straightforward proofs of these types of results.

\section{References}

[1] George E. Andrews. MacMahon's partition analysis. I. The lecture hall partition theorem. In Mathematical essays in honor of Gian-Carlo Rota (Cambridge, MA, 1996), pages 1-22. Birkhäuser Boston, Boston, MA, 1998.

[2] George E. Andrews. MacMahon's Partition Analysis II: Fundamental theorems. Annals of Combinatorics, 4(3-4):327-338, 2000.

[3] George E. Andrews and Peter Paule. MacMahon's partition analysis. IV. Hypergeometric multisums. Sém. Lothar. Combin., 42, 1999. The Andrews Festschrift (Maratea, 1998).

[4] George E. Andrews, Peter Paule, and Axel Riese. MacMahon's Partition Analysis VI: A new reduction theorem. submitted, 2000.

[5] George E. Andrews, Peter Paule, and Axel Riese. MacMahon's Partition Analysis VIII: Plane partitions diamonds. submitted, 2000.

[6] George E. Andrews, Peter Paule, and Axel Riese. MacMahon's Partition Analysis III: The Omega package. European J, Comb., 22(7):887-904, 2001.

[7] George E. Andrews, Peter Paule, and Axel Riese. MacMahon's Partition Analysis IX: $k$-gon partitions. Bull. Austral. Math. Soc., 64(2):321-329, 2001.

[8] George E. Andrews, Peter Paule, and Axel Riese. MacMahon's Partition Analysis VII: Constrained compositions. submitted, 2001.

[9] George E. Andrews, Peter Paule, Axel Riese, and Volker Strehl. MacMahon's partition analysis V: Bijections, recursions, and magic squares. In Algebraic combinatorics and applications (Gößweinstein, 1999), pages 1-39. Springer, Berlin, 2001.

[10] Mireille Bousquet-Mélou and Kimmo Eriksson. Lecture hall partitions. Ramanujan J., 1(1):101$111,1997$.

[11] Mireille Bousquet-Mélou and Kimmo Eriksson. Lecture hall partitions II. Ramanujan J., 1(2):165-185, 1997. 
[12] Rod Canfield, Sylvie Corteel, and Pawel Hitczenko. Random partitions with non negative $r^{\text {th }}$ differences. Adv. Applied Maths, 27:298-317, 2001.

[13] Sylvie Corteel and Carla D. Savage. Anti-lecture hall compositions. Discrete Math., to appear, 2002.

[14] D. R. Hickerson. A partition identity of the Euler type. Amer. Math. Monthly, 81:627-629, 1974.

[15] Percy A. MacMahon. Combinatory analysis. Chelsea Publishing Co., New York, 1960. Two volumes (bound as one).

[16] Richard P. Stanley. Linear homogeneous Diophantine equations and magic labelings of graphs. Duke Math. J., 40:607-632, 1973.

[17] Ae Ja Yee. On combinatorics of lecture hall partitions. Ramanujan Journal, 5:247-262, 2001. Preprint.

[18] Ae Ja Yee. On the refined lecture hall theorem. Discrete Math., 248(1-3):293-298, 2002.

[19] Doron Zeilberger. Sylvie Corteel's one line proof of a partition theorem generated by AndrewsPaule-Riese's computer. Shalosh B. Ekhad's and Doron Zeilberger's Very Own Journal, 1998. 